\title{
On Hilbert modular forms modulo $p$ : explicit ring structure
}

Shoyu Nagaoka

\begin{abstract}
H.P.F. Swinnerton-Dyer determined the structure of the ring of modular forms modulo $p$ in the elliptic modular case. In this paper, the structure of the ring of Hilbert modular forms modulo $p$ is studied. In the case where the discriminant of corresponding quadratic field is 8 (or 5), the explicit structure is determined.
\end{abstract}

\section{Introduction}

In [9] Swinnerton-Dyer determined the structure of the ring of modular forms modulo $p$ in the elliptic modular case. The result has been applied in several fields in the theory of modular forms, for example, the $p$-adic theory of modular forms (e.g. cf. Serre [8]). In this note, we try to generalize the result to the case of symmetric Hilbert modular forms for real quadratic fields of small discriminant. We have already developed a generalization in the Siegel modular case of degree 2, which is important in our proof (cf. Theorem 4.1). A geometric approach has been developed in recent studies by E. Goren (for example, [3] and [4]).

\section{Hilbert modular forms for a real quadratic field}

Let $\mathbb{K}$ be a real quadratic field with the discriminant $d_{\mathbb{K}}$ and the ring of integers $\mathcal{O}_{\mathbb{K}}$. We denote by $\mathbb{H}$ the upper-half plane in $\mathbb{C}$. The Hilbert modular group $\Gamma_{\mathbb{K}}:=S L\left(2, \mathcal{O}_{\mathbb{K}}\right)$ acts on $\mathbb{H}^{2}=\mathbb{H} \times \mathbb{H}$ by

$$
\left(\begin{array}{ll}
a & b \\
c & d
\end{array}\right) \circ\left(z_{1}, z_{2}\right):=\left(\frac{a z_{1}+b}{c z_{1}+d}, \frac{\bar{a} z_{2}+\bar{b}}{\bar{c} z_{2}+\bar{d}}\right),
$$

where $\bar{x}$ denotes the conjugation of $x \in \mathbb{K}$ over $\mathbb{Q}$.

2000 Mathematics Subject Classification: 11F41.

Keywords: Hilbert modular forms, modular forms $\bmod p$. 
Let $A_{\mathbb{C}}\left(\Gamma_{\mathbb{K}}\right)_{k}$ be the complex vector space of symmetric Hilbert modular forms of parallel weight $k$ for $\Gamma_{\mathbb{K}}$. Each element $f(\tau)$ of $A_{\mathbb{C}}\left(\Gamma_{\mathbb{K}}\right)_{k}$ admits a Fourier expansion of the form

$$
f(\tau)=\sum_{0 \ll \nu \in \mathfrak{o}_{\mathbb{K}}^{-1}} a_{f}(\tau) \exp [2 \pi \sqrt{-1} \operatorname{tr}(\nu \tau)],
$$

where $\tau=\left(z_{1}, z_{2}\right) \in \mathbb{H}^{2}, \operatorname{tr}(\nu \tau)=\nu z_{1}+\bar{\nu} z_{2}$ and the summation is extended over the elements $\nu$ in the inverse different $\mathfrak{d}_{\mathbb{K}}^{-1}$ which are semi-totally positive.

From now on, we restrict ourselves to the case

$$
\mathbb{K}=\mathbb{Q}(\sqrt{2}) .
$$

(There is another case $\mathbb{K}=\mathbb{Q}(\sqrt{5})$ where our discussion leads to similar results: cf. section 5 , Remark (2)).

In this case, we have $d_{\mathbb{K}}=8$ and $\mathfrak{d}_{\mathbb{K}}=2 \sqrt{2} \mathcal{O}_{\mathbb{K}}$. We fix an integral basis $\{1, \sqrt{2}\}$ and introduce new variables:

$$
x=: \exp \left[\pi \sqrt{-1}\left(z_{1}-z_{2}\right) / \sqrt{2}\right], q=\exp \left[\pi \sqrt{-1}\left(z_{1}+z_{2}\right)\right] .
$$

Then, the above Fourier expansion is rewritten as

$$
\begin{aligned}
f(\tau)= & \sum_{\nu=(\alpha+\beta \sqrt{2}) / 2 \sqrt{2} \gg 0} a_{f}(\nu) x^{\alpha} q^{\beta} \\
= & a_{f}(0)+a_{f}((-1+\sqrt{2}) / 2 \sqrt{2}) x^{-1} q+a_{f}(1 / 2) q+a_{f}((1+\sqrt{2}) / 2 \sqrt{2}) x q \\
& +a_{f}((-2+2 \sqrt{2}) / 2 \sqrt{2}) x^{-2} q^{2}+a_{f}((-1+2 \sqrt{2}) / 2 \sqrt{2}) x^{-1} q^{2}+a_{f}(1) q^{2} \\
& +a_{f}((1+2 \sqrt{2}) / 2 \sqrt{2}) x q^{2}+a_{f}((2+2 \sqrt{2}) / 2 \sqrt{2}) x^{2} q^{2}+\cdots .
\end{aligned}
$$

By semi-positivity of $\nu$, we may regard $f$ as an element of formal power series ring $\mathbb{C}\left[x^{-1}, x\right] \llbracket q \rrbracket$. For a subring $R$ in $\mathbb{C}$,

$$
A_{R}\left(\Gamma_{\mathbb{K}}\right)_{k}:=\left\{f \in A_{\mathbb{C}}\left(\Gamma_{\mathbb{K}}\right)_{k} \mid a_{f}(\nu) \in R \text { for all } \nu\right\} \subset R\left[x^{-1}, x\right] \llbracket q \rrbracket
$$

and

$$
A_{R}^{(m)}\left(\Gamma_{\mathbb{K}}\right):=\bigoplus_{k \geq 0} A_{R}\left(\Gamma_{\mathbb{K}}\right)_{k m}
$$

For an even positive integer $k$, we can define the normalized Eisenstein series of weight $k$ for $\Gamma_{\mathbb{K}}$ whose Fourier expansion is

$$
G_{k}(\tau)=1+\kappa_{k} \sum_{\substack{\nu \in \mathfrak{d}_{\mathbb{K}}^{-1} \\ 0 \neq \nu \gg 0}} \sigma_{k-1}(\nu) \exp [2 \pi \sqrt{-1} \operatorname{tr}(\nu \tau)]
$$


where

$$
\begin{aligned}
& \kappa_{k}:=\zeta_{\mathbb{K}}(k)^{-1} \cdot(2 \pi)^{2 k} \cdot[(k-1) !]^{-2} \cdot d_{\mathbb{K}}^{1 / 2-k}, \\
& \sigma_{k-1}(\nu):=\sum_{(\nu) \mathfrak{d}_{\mathbb{K}} \subset \mathfrak{b}}|N(\mathfrak{b})|^{k-1} .
\end{aligned}
$$

Since $\kappa_{k} \in \mathbb{Q}$, we have $G_{k} \in A_{\mathbb{Q}}\left(\Gamma_{\mathbb{K}}\right)_{k}$.

Let $E_{k}(z)$ be the normalized Eisenstein series of weight $k$ for $S L(2, \mathbb{Z})$, and let $\Delta(z)$ be a cusp form defined by

$$
\Delta(z)=2^{-6} \cdot 3^{-3}\left(E_{4}^{3}(z)-E_{6}^{2}(z)\right) .
$$

It is well-known that

$$
E_{k} \in A_{\mathbb{Q}}(S L(2, \mathbb{Z}))_{k} \quad \text { and } \quad \Delta \in A_{\mathbb{Z}}(S L(2, \mathbb{Z}))_{12} .
$$

For a function $f\left(\left(z_{1}, z_{2}\right)\right)$ on $\mathbb{H}^{2}$, we define a function on $\mathbb{H}$ by

$$
\mathbb{D}(f)(z):=f((z, z)) .
$$

By the definiton of Hilbert modular form, we see that the map $\mathbb{D}$ induces an $R$-linear map

$$
\mathbb{D}: A_{R}\left(\Gamma_{\mathbb{K}}\right)_{k} \longrightarrow A_{R}(S L(2, \mathbb{Z}))_{2 k} .
$$

In fact, if

$$
f(\tau)=\sum a_{f}(\nu) \exp [2 \pi \sqrt{-1} \operatorname{tr}(\nu \tau)]
$$

in $A_{R}\left(\Gamma_{\mathbb{K}}\right)_{k}$, then the Fourier expansion of $\mathbb{D}(f)$ is

$$
\mathbb{D}(f)(z)=\sum_{n=0}^{\infty} c_{f}(n) \exp [2 \pi \sqrt{-1} n z], c_{f}(n)=\sum_{\operatorname{tr}(\nu)=n} a_{f}(\nu) .
$$

Put

$$
\begin{aligned}
H_{2}:= & G_{2} \\
= & 1+2^{4} \cdot 3\left\{\left(x^{-1}+3+x\right) q+\right. \\
& \left.\quad+\left(7 x^{-2}+8 x^{-1}+15+8 x+7 x^{2}\right) q^{2}+\cdots\right\}, \\
H_{4}:= & 2^{-6} \cdot 3^{-2} \cdot 11\left(G_{2}^{2}-G_{4}\right) \\
= & \left(x^{-1}-2+x\right) q+\left(-4 x^{-2}-8 x^{-1}+24-8 x-4 x^{2}\right) q^{2} \cdots, \\
H_{6}:= & -2^{-8} \cdot 3^{-3} \cdot 13^{-1} \cdot 5 \cdot 7^{2} G_{2}^{3}-2^{-7} \cdot 3^{-3} \cdot 5^{-1} \cdot 13^{-1} 19^{2} G_{6} \\
& +2^{-8} \cdot 3^{-2} \cdot 5^{-1} \cdot 13^{-1} \cdot 11 \cdot 59 G_{2} G_{4} \\
= & q+\left(-2 x^{-2}-16 x^{-1}+12-16 x-2 x^{2}\right) q^{2}+\cdots .
\end{aligned}
$$


Proposition 2.1 Let $\mathbb{Z}_{(p)}$ be the local ring at $p$ ( $p$ : prime).

(1) $H_{k} \in A_{\mathbb{Z}}\left(\Gamma_{\mathbb{K}}\right)_{k} \subset A_{\mathbb{Z}_{(p)}}\left(\Gamma_{\mathbb{K}}\right)_{k} \quad(k=2,4,6)$ and

$$
\mathbb{D}\left(H_{2}\right)=E_{4}, \mathbb{D}\left(H_{4}\right)=0, \mathbb{D}\left(H_{6}\right)=\Delta .
$$

(2) If $f \in A_{\mathbb{Z}_{(p)}}\left(\Gamma_{\mathbb{K}}\right)_{k}$, $(k:$ even $)$, then there exists a polynomial $P\left(X_{1}, X_{2}, X_{3}\right) \in \mathbb{Z}_{(p)}\left[X_{1}, X_{2}, X_{3}\right]$ satisfying

$$
f=P\left(H_{2}, H_{4}, H_{6}\right) .
$$

Namely,

$$
A_{\mathbb{Z}_{(p)}}^{(2)}\left(\Gamma_{\mathbb{K}}\right)=\mathbb{Z}_{(p)}\left[H_{2}, H_{4}, H_{6}\right]
$$

Proposition 2.2 ([6, Propositions 3.1, 3.2])

(1) There exists an odd weight form $\mathrm{H}_{9}$ with integral Fourier coefficients:

$$
H_{9}=q-\left(96^{-1} x+336+96 x\right) q^{2}+\cdots \in A_{\mathbb{Z}}\left(\Gamma_{\mathbb{K}}\right)_{9} \subset A_{\mathbb{Z}_{(p)}}\left(\Gamma_{\mathbb{K}}\right)_{9} .
$$

(2) If $k$ is odd, then $A_{\mathbb{Z}_{(p)}}\left(\Gamma_{\mathbb{K}}\right)_{k}=H_{9} \cdot A_{\mathbb{Z}_{(p)}}\left(\Gamma_{\mathbb{K}}\right)_{k-9}$.

(3) $H_{9}^{2}$ has a polynomial expression in $H_{2}, H_{4}$, and $H_{6}$ :

$$
H_{9}^{2}=H_{2}^{3} H_{6}^{2}+2^{2} H_{2}^{2} H_{4}^{2} H_{6}-2^{5} \cdot 3^{2} H_{2} H_{4} H_{6}^{2}-2^{10} H_{4}^{3} H_{6}-2^{6} \cdot 3^{3} H_{6}^{3} .
$$

\section{Siegel modular form and modular embedding}

Let $A_{\mathbb{C}}\left(\Gamma_{\mathbb{K}}\right)_{k}$ be the complex vector space of Siegel modular forms of weight $k$ for $\Gamma_{n}:=S p(n, \mathbb{Z})$. As is well known, each element $F(Z)$ in $A_{\mathbb{C}}\left(\Gamma_{n}\right)_{k}$ admits a Fourier expansion of the form

$$
F(Z)=\sum_{T \geq 0} a_{F}(T) \exp [2 \pi \sqrt{-1} \operatorname{tr}(T Z)], Z \in \mathbb{H}_{n}
$$

where $\mathbb{H}_{n}$ is the Siegel upper-half space of degree $n$ and the summation is extended over all half-integral, positive semi-definite, symmetric matrices of degree $n$. As in the previous case, we can define an $R$-module $A_{R}\left(\Gamma_{n}\right)_{k}$.

We now introduce a modular embedding from $A_{\mathbb{C}}\left(\Gamma_{\mathbb{K}}\right)_{k}(\mathbb{K}=\mathbb{Q}(\sqrt{2}))$ to $A_{\mathbb{C}}\left(\Gamma_{2}\right)_{k}$.

We fix a fundamental unit $\varepsilon=1+\sqrt{2}$ in $\mathcal{O}_{\mathbb{K}}$ and define a matrix $A$ by

$$
A=\left(\begin{array}{cc}
\alpha & \bar{\alpha} \\
\bar{\alpha} & -\alpha
\end{array}\right), \alpha=\sqrt{\varepsilon / 2 \sqrt{2}}, \bar{\alpha}=\sqrt{-\bar{\varepsilon} / 2 \sqrt{2}}
$$


First, we define a mapping $\Phi: \mathbb{H}^{2}=\mathbb{H} \times \mathbb{H} \longrightarrow \mathbb{H}_{2}$ by

$$
\begin{aligned}
\Phi(\tau) & =\Phi\left(\left(z_{1}, z_{2}\right)\right):=A\left(\begin{array}{cc}
z_{1} & 0 \\
0 & z_{2}
\end{array}\right) A \\
& =\left(\begin{array}{cc}
\operatorname{tr}((\varepsilon / 2 \sqrt{2}) \tau) & \operatorname{tr}((1 / 2 \sqrt{2}) \tau) \\
\operatorname{tr}((1 / 2 \sqrt{2}) \tau) & \operatorname{tr}((-\bar{\varepsilon} / 2 \sqrt{2}) \tau)
\end{array}\right) .
\end{aligned}
$$

Secondly, we define a mapping $\Psi: \Gamma_{\mathbb{K}}=S L\left(2, \mathcal{O}_{\mathbb{K}}\right) \longrightarrow \Gamma_{2}=S p(2, \mathbb{Z})$ by

$$
\begin{aligned}
\Psi\left(\left(\begin{array}{ll}
a & b \\
c & d
\end{array}\right)\right) & =\Psi\left(\left(\begin{array}{cccc}
a_{1}+a_{2} \sqrt{2} & b_{1}+b_{2} \sqrt{2} \\
c_{1}+c_{2} \sqrt{2} & d_{1}+d_{2} \sqrt{2}
\end{array}\right)\right) \\
& =\left(\begin{array}{cccc}
a_{1}+a_{2} & a_{2} & b_{1}+b_{2} & b_{2} \\
a_{2} & a_{1}-a_{2} & b_{2} & b_{1}-b_{2} \\
c_{1}+c_{2} & c_{2} & d_{1}+d_{2} & d_{2} \\
c_{2} & c_{1}-c_{2} & d_{2} & d_{1}-d_{2}
\end{array}\right) .
\end{aligned}
$$

Proposition 3.1 ([6, Proposition 2.1]) If $F$ is a Siegel modular form in $A_{\mathbb{C}}\left(\Gamma_{2}\right)_{k}$, then $\Phi(F)=F \circ \Phi$ is a symmetric Hilbert modular form in $A_{\mathbb{C}}\left(\Gamma_{\mathbb{K}}\right)_{k}$.

We calculate the Fourier coefficient of $\Phi(F)$. Set

$$
F(Z)=\sum_{T \geq 0} a_{F}(T) \exp [2 \pi \sqrt{-1} \operatorname{tr}(T Z)]
$$

We take a half-integral, positive semi-definite matrix

$$
T=\left(\begin{array}{cc}
m & l / 2 \\
l / 2 & n
\end{array}\right), \quad(m, n, l \in \mathbb{Z})
$$

Since

$$
\exp [2 \pi \sqrt{-1} \operatorname{tr}(T \Phi(\tau))]=x^{m-n+l} q^{m+n}
$$

we have

$$
\Phi(F)(\tau)=\sum_{(\alpha+\beta \sqrt{2}) / 2 \sqrt{2} \gg 0}\left(\sum_{\substack{m-n+l=\alpha \\
m+n=\beta}} a_{F}\left(\left(\begin{array}{cc}
m & l / 2 \\
l / 2 & n
\end{array}\right)\right)\right) x^{\alpha} q^{\beta} .
$$

Corollary 3.1 Let $R$ be a subring of $\mathbb{C}$. If $F \in A_{R}\left(\Gamma_{2}\right)_{k}$, then

$$
\Phi(F) \in A_{R}\left(\Gamma_{\mathbb{K}}\right)_{k}
$$




\section{Hilbert modular form modulo $p$}

As before, $p$ be a prime number, and let $\mathbb{Z}_{(p)}$ be the local ring at $p$. We set

$$
\begin{aligned}
A_{\mathbb{F}_{p}}\left(\Gamma_{\mathbb{K}}\right)_{k} & :=\left\{\tilde{f}=\sum \widetilde{a_{f}(\nu)} x^{\alpha} q^{\beta} \mid f \in A_{\mathbb{Z}_{(p)}}\left(\Gamma_{\mathbb{K}}\right)_{k}\right\} \\
& \subset \mathbb{F}_{p}\left[x^{-1}, x\right] \llbracket q \rrbracket
\end{aligned}
$$

where the tilde denotes the reduction modulo $p$. Let $A_{\mathbb{F}_{p}}^{(m)}\left(\Gamma_{\mathbb{K}}\right)$ denote the subring of $\mathbb{F}_{p}\left[x^{-1}, x\right] \llbracket q \rrbracket$ generated by $A_{\mathbb{F}_{p}}\left(\Gamma_{\mathbb{K}}\right)_{k}$ for $k=0, m, 2 m, 3 m, \cdots$. The first theorem is as follows.

Theorem 4.1 (Existence Theorem) Assume that $\mathbb{K}=\mathbb{Q}(\sqrt{2})$ and $p \geq 3$. Then, there exists a Hilbert modular form $f_{p-1} \in A_{\mathbb{Z}_{(p)}}\left(\Gamma_{\mathbb{K}}\right)_{p-1}$ satisfying

$$
f_{p-1} \equiv 1 \quad(\bmod p)
$$

where the congruence is the Fourier coefficientwise congruence.

Proof. First assume that $p \geq 5$. By [7, Theorem A] there exists a Siegel modular form $F_{p-1} \in A_{\mathbb{Z}_{(p)}}\left(\Gamma_{2}\right)_{p-1}$ satisfying

$$
F_{p-1} \equiv 1 \quad(\bmod p) .
$$

If we set

$$
f_{p-1}:=\Phi\left(F_{p-1}\right)
$$

then, by (3.3), we see that

$$
f_{p-1} \in A_{\mathbb{Z}_{(p)}}\left(\Gamma_{\mathbb{K}}\right)_{p-1}
$$

has the desired property. When $p=3$, we can take $f_{p-1}=f_{2}=G_{2}$.

Remark. In the original (elliptic modular) case, it is easy to find such modular form: the weight $p-1$ Eisenstein series $E_{p-1}$ satisfies $E_{p-1} \equiv 1$ $(\bmod p)$. However, the Hilbert-Eisenstein series $G_{p-1}$ does not satisfy the congruence $G_{p-1} \equiv 1(\bmod p)$ in general. In fact, $G_{12} \not \equiv 1(\bmod 13)$ (for example, [3, p. 373]).

In the following we shall determine the structure of $A_{\mathbb{F}_{p}}^{(2)}\left(\Gamma_{\mathbb{K}}\right)$ under the condition

$$
p \equiv 3 \quad(\bmod 4)
$$


Theorem 4.2 Assume that $\mathbb{K}=\mathbb{Q}(\sqrt{2})$.

(1) If $p \geq 5$ is a prime number such that $p \equiv 3(\bmod 4)$, then

$$
A_{\mathbb{F}_{p}}^{(2)}\left(\Gamma_{\mathbb{K}}\right) \cong \mathbb{F}_{p}\left[\tilde{H}_{2}, \tilde{H}_{4}, \tilde{H}_{6}\right] /\left(\tilde{A}_{p}\left(\tilde{H}_{2}, \tilde{H}_{4}, \tilde{H}_{6}\right)-1\right)
$$

where $H_{2}, H_{4}$, and $H_{6}$ are generators of $A_{\mathbb{Z}_{(p)}}^{(2)}\left(\Gamma_{\mathbb{K}}\right)$ (cf. Proposition 2.1) and $A_{p}\left(X_{1}, X_{2}, X_{3}\right) \in \mathbb{Z}_{(p)}\left[X_{1}, X_{2}, X_{3}\right]$ is a polynomial defined by

$$
f_{p-1}=A_{p}\left(H_{2}, H_{4}, H_{6}\right) .
$$

(2) If $p=2$ or 3 , then

$$
A_{\mathbb{F}_{p}}^{(2)}\left(\Gamma_{\mathbb{K}}\right) \cong \mathbb{F}_{p}\left[\tilde{H}_{4}, \tilde{H}_{6}\right]
$$

Proof. (1) We recall the identities

$$
\mathbb{D}\left(H_{2}\right)=E_{4}, \quad \mathbb{D}\left(H_{4}\right)=0, \quad \mathbb{D}\left(H_{6}\right)=\Delta
$$

and consider the following diagram:

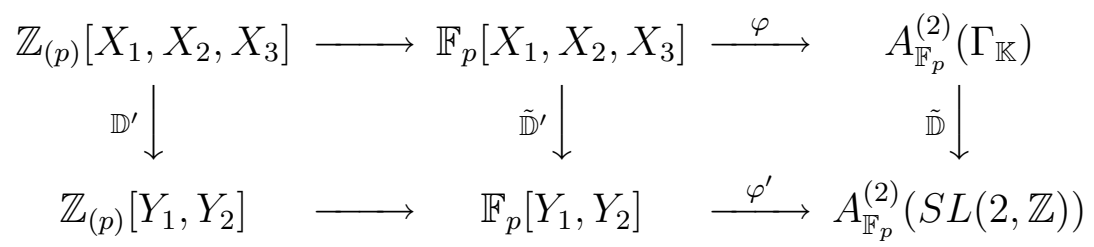

where

$$
\begin{array}{ll}
\varphi: & \varphi\left(\tilde{P}\left(X_{1}, X_{2}, X_{3}\right)\right):=\tilde{P}\left(\tilde{H}_{2}, \tilde{H}_{4}, \tilde{H}_{6}\right) . \\
\varphi^{\prime}: & \varphi^{\prime}\left(\tilde{Q}\left(Y_{1}, Y_{2}\right)\right):=\tilde{Q}\left(\tilde{E}_{4}, \tilde{E}_{6}\right) . \\
\mathbb{D}^{\prime}: & \mathbb{D}^{\prime}\left(P\left(X_{1}, X_{2}, X_{3}\right)\right):=P\left(Y_{1}, 0,2^{-6} \cdot 3^{-3}\left(Y_{1}^{3}-Y_{2}^{2}\right)\right) . \\
\tilde{\mathbb{D}}^{\prime}: & \tilde{\mathbb{D}^{\prime}}\left(\tilde{P}\left(X_{1}, X_{2}, X_{3}\right)\right):=\tilde{P}\left(Y_{1}, 0, \tilde{a}\left(Y_{1}^{3}-Y_{2}^{2}\right)\right), \quad \tilde{a}=2^{-6} \cdot 3^{-3} \bmod p . \\
\tilde{\mathbb{D}}: & \tilde{\mathbb{D}}(\tilde{f})=\widetilde{\mathbb{D}(f)}, \quad \tilde{f}=\sum \widetilde{a_{f}(\nu)} x^{\alpha} q^{\beta} \in A_{\mathbb{F}_{p}}\left(\Gamma_{\mathbb{K}}\right) \subset \mathbb{F}_{p}\left[x^{-1}, x\right] \llbracket q \rrbracket .
\end{array}
$$

By Proposition 2.1,(2), the map $\varphi$ is surjective. For the Hilbert modular form $f_{p-1}$, we represent it as a polynomial in $H_{2}, H_{4}$, and $H_{6}$

$$
f_{p-1}=A_{p}\left(H_{2}, H_{4}, H_{6}\right), A_{p}\left(X_{1}, X_{2}, X_{3}\right) \in \mathbb{Z}_{(p)}\left[X_{1}, X_{2}, X_{3}\right] .
$$

The congruence $f_{p-1} \equiv 1(\bmod p)$ implies $\tilde{A}_{p}-1 \in \operatorname{Ker} \varphi$. Therefore it suffices to show that

$$
\operatorname{Ker} \varphi=\left(\tilde{A}_{p}-1\right) \quad(\text { principal ideal }) .
$$


To prove this, we first note that

$$
\operatorname{Im} \tilde{\mathbb{D}}=A_{\mathbb{F}_{p}}^{(4)}(S L(2, \mathbb{Z})) \subset A_{\mathbb{F}_{p}}^{(2)}(S L(2, \mathbb{Z}))
$$

and

$$
\text { Krull } \operatorname{dim} A_{\mathbb{F}_{p}}^{(4)}(S L(2, \mathbb{Z}))=\text { Krull } \operatorname{dim} A_{\mathbb{F}_{p}}^{(2)}(S L(2, \mathbb{Z}))=1 .
$$

The first identity in the second formula comes from the fact that $\tilde{E}_{6}$ is integral over $A_{\mathbb{F}_{p}}^{(4)}(S L(2, \mathbb{Z}))$. Since Ker $\tilde{\mathbb{D}} \neq 0$ (for example, $\left.0 \neq \tilde{H}_{4} \in \operatorname{Ker} \tilde{\mathbb{D}}\right)$, we have

$$
\text { Krull } \operatorname{dim} A_{\mathbb{F}_{p}}^{(2)}\left(\Gamma_{\mathbb{K}}\right)=2
$$

Hence, the irreducibility of $\tilde{A}_{p}-1$ implies our statement:

$$
A_{\mathbb{F}_{p}}^{(2)}\left(\Gamma_{\mathbb{K}}\right) \cong \mathbb{F}_{p}\left[\tilde{H}_{2}, \tilde{H}_{4}, \tilde{H}_{6}\right] /\left(\tilde{A}_{p}\left(\tilde{H}_{2}, \tilde{H}_{4}, \tilde{H}_{6}\right)-1\right)
$$

We shall show the irreducibility under the condition $p \equiv 3(\bmod 4)$. For this purpose, we recall the corresponding fact in the elliptic modular case. The normalized Eisenstein series $E_{p-1}$ satisfies $E_{p-1} \equiv 1(\bmod p)$. Moreover, if we represent $E_{p-1}$ as

$$
E_{p-1}=B_{p}\left(E_{4}, E_{6}\right) \quad \text { with } B_{p}\left(Y_{1}, Y_{2}\right) \in \mathbb{Z}_{(p)}\left[Y_{1}, Y_{2}\right],
$$

then $B_{p}\left(Y_{1}, Y_{2}\right)-1$ is irreducible in $\mathbb{F}_{p}\left[Y_{1}, Y_{2}\right]$ (cf. [9]). From this fact, we get the decomposition

$$
\tilde{\mathbb{D}}^{\prime}\left(\tilde{A}_{p}\left(X_{1}, X_{2}, X_{3}\right)-1\right)=\left(\tilde{B}_{p}\left(Y_{1}, Y_{2}\right)+1\right)\left(\tilde{B}_{p}\left(Y_{1}, Y_{2}\right)-1\right) .
$$

Here, we note that both factors $\tilde{B}_{p}+1$ and $\tilde{B}_{p}-1$ are irreducible. Now we assume that $\tilde{A}_{p}-1$ is reducible. Then, the shape of the decomposition must be

$$
\tilde{A}_{p}-1=\left(\tilde{G}^{(a)}+\tilde{G}^{(a-1)}+\cdots+\tilde{G}^{(0)}\right)\left(\tilde{H}^{(a)}+\tilde{H}^{(a-1)}+\cdots+\tilde{H}^{(0)}\right),
$$

where $G^{(j)}$ (also $\left.H^{(j)}\right)$ is a polynomial consisting of terms such as

$$
a_{\alpha \beta \gamma} X_{1}^{\alpha} X_{2}^{\beta} X_{3}^{\gamma}
$$

with $2 \alpha+4 \beta+6 \gamma=j$, namely, terms of isobaric degree $j$. Combining (4.1) and (4.2), we have $2 a=p-1$. Since $a$ is even, the prime $p$ must be congruent to one modulo 4. This contradicts our assumption. (2) If $p=2$ or 3 , then $\tilde{H}_{2}=1$. Moreover, $\tilde{H}_{4}$ and $\tilde{H}_{6}$ are algebraically independent because the Fourier expansion of $H_{4}$ (resp. $\left.H_{6}\right)$ starts at the term $\left(x^{-1}-2+x\right) q$ (resp.q). 
From the above result and Proposition 2.2, we can easily determine the structure of the whole ring $A_{\mathbb{F}_{p}}\left(\Gamma_{\mathbb{K}}\right)=A_{\mathbb{F}_{p}}^{(1)}\left(\Gamma_{\mathbb{K}}\right)$.

Set

$$
\begin{aligned}
C\left(X_{1}, X_{2}, X_{3}, X_{4}\right):= & X_{1}^{3} X_{3}^{2}+2^{2} X_{1}^{2} X_{2}^{2} X_{3}-2^{5} \cdot 3^{2} X_{1} X_{2} X_{3}^{2}-2^{10} X_{2}^{3} X_{3} \\
& -2^{6} \cdot 3^{3} X_{3}^{3}-X_{4}^{2} \in \mathbb{Z}\left[X_{1}, X_{2}, X_{3}, X_{4}\right]
\end{aligned}
$$

It should be noted that the polynomial is chosen as

$$
C\left(H_{2}, H_{4}, H_{6}, H_{9}\right)=0, \quad(\text { cf. }(2.3)) .
$$

Let $\tilde{C}_{p}\left(X_{1}, X_{2}, X_{3}, X_{4}\right) \in \mathbb{F}_{p}\left[X_{1}, X_{2}, X_{3}, X_{4}\right]$ be the reduction modulo $p$. Combining this and Theorem 4.2, we obtain the following:

Theorem 4.3 Assume that $\mathbb{K}=\mathbb{Q}(\sqrt{2})$.

(1) If $p \geq 5$ and $p \equiv 3(\bmod 4)$, then

$$
A_{\mathbb{F}_{p}}\left(\Gamma_{\mathbb{K}}\right) \cong \mathbb{F}_{p}\left[\tilde{H}_{2}, \tilde{H}_{4}, \tilde{H}_{6}, \tilde{H}_{9}\right] /\left(\tilde{A}_{p}\left(\tilde{H}_{2}, \tilde{H}_{4}, \tilde{H}_{6}\right)-1, \tilde{C}_{p}\left(\tilde{H}_{2}, \tilde{H}_{4}, \tilde{H}_{6}, \tilde{H}_{9}\right)\right) .
$$

(2) If $p=2$ or 3 ,

$$
A_{\mathbb{F}_{p}}\left(\Gamma_{\mathbb{K}}\right) \cong \mathbb{F}_{p}\left[\tilde{H}_{4}, \tilde{H}_{6}, \tilde{H}_{9}\right] /\left(\tilde{C}_{p}\right)
$$

that is

$$
\begin{aligned}
& A_{\mathbb{F}_{3}}\left(\Gamma_{\mathbb{K}}\right) \cong \mathbb{F}_{3}\left[\tilde{H}_{4}, \tilde{H}_{6}, \tilde{H}_{9}\right] /\left(\tilde{H}_{6}^{2}+\tilde{H}_{4}^{2} \tilde{H}_{6}+2 \tilde{H}_{6}+2 \tilde{H}_{9}^{2}\right), \\
& A_{\mathbb{F}_{2}}\left(\Gamma_{\mathbb{K}}\right)=\mathbb{F}_{2}\left[\tilde{H}_{4}, \tilde{H}_{6}\right]=\mathbb{F}_{2}\left[\tilde{H}_{4}, \tilde{H}_{9}\right]
\end{aligned}
$$

\section{Remark}

(1) Case $p \equiv 1(\bmod 4)$ :

In the above discussion, the result was restricted to the case $p \equiv 3$ $(\bmod 4)$. What about the case $p \equiv 1(\bmod 4)$ ? In this case also, the irreducibility of $\tilde{A}_{p}-1$ produces similar results. The first few examples show the irreducibility.

$$
\begin{aligned}
& p=5: \quad \tilde{A}_{5}-1=X_{1}^{2}+4 X_{2}-1, \quad \mathbb{D}^{\prime}\left(\tilde{A}_{5}-1\right)=Y_{1}^{2}-1, \quad \tilde{B}_{5}-1=Y_{1}-1 . \\
& p=7: \tilde{A}_{7}-1=X_{1}^{3}+3 X_{1} X_{2}+X_{3}-1, \quad \mathbb{D}^{\prime}\left(\tilde{A}_{7}-1\right)=Y_{2}^{2}-1, \\
& \tilde{B}_{7}-1=Y_{2}-1 . \\
& p=11: \tilde{A}_{11}-1=X_{1}^{5}+2 X_{1}^{3} X_{2}+10 X_{1}^{2} X_{3}+X_{1} X_{2}^{2}+X_{2} X_{3}-1, \\
& \mathbb{D}^{\prime}\left(\tilde{A}_{11}-1\right)=Y_{1}^{2} Y_{2}^{2}-1, \quad \tilde{B}_{11}-1=Y_{1} Y_{2}-1 . \\
& p=13: \tilde{A}_{13}-1=X_{1}^{6}+11 X_{1}^{4} X_{2}+3 X_{1}^{3} X_{3}+11 X_{1}^{2} X_{2}^{2}+2 X_{1} X_{2} X_{3}+10 X_{2}^{3} \\
& \quad+12 X_{3}^{2}-1, \\
& \mathbb{D}^{\prime}\left(\tilde{A}_{13}-1\right)=10 Y_{1}^{6}+5 Y_{1}^{3} Y_{2}^{2}+12 Y_{2}^{4}-1, \quad \tilde{B}_{13}-1=6 Y_{1}^{3}+8 Y_{2}^{2}-1 .
\end{aligned}
$$


(2) Case for $\mathbb{K}=\mathbb{Q}(\sqrt{5})$ :

The proposed method is applicable for the case $\mathbb{K}=\mathbb{Q}(\sqrt{5})$. In this paper, we present the statement without proof.

Let $G_{k}$ be the normalized Eisenstein series of weight $k$ for $\Gamma_{\mathbb{Q}(\sqrt{5})}$. We define four modular forms $J_{k}(k=2,6,1012)$ as follows:

$$
\begin{aligned}
J_{2} & :=G_{2} \\
& =1+2^{3} \cdot 3 \cdot 5\left\{\left(x^{-1}+x\right) q+\left(x^{-4}+5 x^{-2}+6+5 x^{2}+x^{4}\right) q^{2}+\cdots\right\}, \\
J_{6} & :=2^{-5} \cdot 3^{-3} \cdot 5^{-2} \cdot 67\left(G_{2}^{3}-G_{6}\right) \\
& =\left(x^{-1}+x\right) q+\left(x^{-4}+20 x^{-2}-90+20 x^{2}+x^{4}\right) q^{2} \cdots, \\
J_{10} & :=2^{-10} \cdot 3^{-5} \cdot 5^{-5} \cdot 7^{-1}\left(412751 G_{10}-5 \cdot 67 \cdot 2293 G_{2}^{2} G_{6}+2^{2} \cdot 3 \cdot 7 \cdot 4231 G_{2}^{5}\right) \\
& =\left(x^{-1}-x\right)^{2} q^{2}-2\left(x^{-1}-x\right)\left(x^{-4}+10 x^{-2}-10 x^{2}-x^{4}\right) q^{3}+\cdots, \\
J_{12} & :=2^{-2}\left(J_{6}^{2}-J_{2} J_{10}\right) \\
& =q^{2}+\left(x^{-5}-15 x^{-3}-10 x^{-1}-10 x-15 x^{3}+x^{5}\right) q^{3}+\cdots,
\end{aligned}
$$

where $x=\exp \left[\pi \sqrt{-1}\left(z_{1}-z_{2}\right) / \sqrt{5}\right], q=\exp \left[\pi \sqrt{-1}\left(z_{1}+z_{2}\right)\right]$.

Theorem 5.1 (Existence Theorem) Assume that $\mathbb{K}=\mathbb{Q}(\sqrt{5})$ and $p \geq 3$. Then, there exists a Hilbert modular form $f_{p-1} \in A_{\mathbb{Z}_{(p)}}\left(\Gamma_{\mathbb{K}}\right)_{p-1}$ satisfying

$$
f_{p-1} \equiv 1 \quad(\bmod p) .
$$

Theorem 5.2 Assume that $\mathbb{K}=\mathbb{Q}(\sqrt{5})$.

(1) If $p \geq 5$ is a prime number such that $p \equiv 3(\bmod 4)$, then

$$
A_{\mathbb{F}_{p}}^{(2)}\left(\Gamma_{\mathbb{K}}\right) \cong \mathbb{F}_{p}\left[\tilde{J}_{2}, \tilde{J}_{6}, \tilde{J}_{10}\right] /\left(\tilde{A}_{p}\left(\tilde{J}_{2}, \tilde{J}_{6}, \tilde{J}_{10}\right)-1\right)
$$

where $J_{2}, J_{6}, J_{10}$ are generators of $A_{\mathbb{Z}_{(p)}^{(2)}}^{\left(\Gamma_{\mathbb{K}}\right)}$ and

$$
A_{p}\left(X_{1}, X_{2}, X_{3}\right) \in \mathbb{Z}_{(p)}\left[X_{1}, X_{2}, X_{3}\right]
$$

is a polynomial defined by

$$
f_{p-1}=A_{p}\left(J_{2}, J_{6}, J_{10}\right) .
$$

$$
\begin{aligned}
A_{\mathbb{F}_{3}}^{(2)}\left(\Gamma_{\mathbb{K}}\right) & =\mathbb{F}_{3}\left[\tilde{J}_{6}, \tilde{J}_{10}\right] . \\
A_{\mathbb{F}_{2}}^{(2)}\left(\Gamma_{\mathbb{K}}\right) & \cong \mathbb{F}_{2}\left[\tilde{J}_{6}, \tilde{J}_{10}, \tilde{J}_{12}\right] /\left(\tilde{J}_{6}^{2}+\tilde{J}_{10}^{2}\right)
\end{aligned}
$$


Proposition 5.1 ([6, Theorem 3.1 and Proposition 3.3])

(1) There exists an odd weight form $J_{15}$ with integral Fourier coefficients:

$$
J_{15}=q^{2}-\left(x^{-5}+275 x^{-1}+275 x+x^{5}\right) q^{3}+\cdots \in A_{\mathbb{Z}}\left(\Gamma_{\mathbb{K}}\right)_{15} \subset A_{\mathbb{Z}_{(p)}}\left(\Gamma_{\mathbb{K}}\right)_{15} .
$$

(2) If $k$ is odd, then $A_{\mathbb{Z}_{(p)}}\left(\Gamma_{\mathbb{K}}\right)_{k}=J_{15} \cdot A_{\mathbb{Z}_{(p)}}\left(\Gamma_{\mathbb{K}}\right)_{k-15}$.

(3) $J_{15}^{2}$ has the following polynomial expressions:

$$
\begin{aligned}
J_{15}^{2}= & 5^{5} J_{10}^{3}-2 \cdot 3^{3} J_{6}^{5}+2 \cdot 5^{2} J_{2} J_{6}^{3} J_{10}+2 \cdot 5^{3} J_{2} J_{6} J_{10} J_{12}+J_{2}^{3} J_{12}^{2} \\
= & 5^{5} J_{10}^{3}-2 \cdot 3^{3} J_{6}^{5}+2^{-1} \cdot 3^{2} \cdot 5^{2} J_{2} J_{6}^{3} J_{10}-2^{-1} \cdot 5^{3} J_{2}^{2} J_{6} J_{10}^{2} \\
& +2^{-4} J_{2}^{3} J_{6}^{4}-2^{-3} J_{2}^{4} J_{6}^{2} J_{10}+2^{-4} J_{2}^{5} J_{10}^{2} .
\end{aligned}
$$

Set

$$
\begin{aligned}
C\left(X_{1}, X_{2}, X_{3}, X_{4}\right):= & X_{4}^{2}-5^{5} X_{3}^{3}+2 \cdot 3^{3} X_{2}^{5}-2^{-1} \cdot 3^{2} \cdot 5^{2} X_{1} X_{2}^{3} X_{3} \\
& +2^{-1} \cdot 5^{3} X_{1}^{2} X_{2} X_{3}^{2}-2^{-4} X_{1}^{3} X_{2}^{4}+2^{-3} X_{1}^{4} X_{2}^{2} X_{3} \\
& -2^{-4} X_{1}^{5} X_{3}^{2} \in \mathbb{Q}\left[X_{1}, X_{2}, X_{3}, X_{4}\right]
\end{aligned}
$$

If $p \neq 2$, then $C\left(X_{1}, X_{2}, X_{3}, X_{4}\right) \in \mathbb{Z}_{(p)}\left[X_{1}, X_{2}, X_{3}, X_{4}\right]$. Denote by $\tilde{C}_{p}\left(X_{1}, X_{2}, X_{3}, X_{4}\right) \in \mathbb{F}_{p}\left[X_{1}, X_{2}, X_{3}, X_{4}\right]$ the reduction modulo $p(p \neq 2)$.

Theorem 5.3 Assume that $\mathbb{K}=\mathbb{Q}(\sqrt{5})$.

(1) If $p \geq 5$ is a prime number such that $p \equiv 3(\bmod 4)$, then

$$
A_{\mathbb{F}_{p}}\left(\Gamma_{\mathbb{K}}\right) \cong \mathbb{F}_{p}\left[\tilde{J}_{2}, \tilde{J}_{6}, \tilde{J}_{10}, \tilde{J}_{15}\right] /\left(\tilde{A}_{p}\left(\tilde{J}_{2}, \tilde{J}_{6}, \tilde{J}_{10}\right)-1, \tilde{C}_{p}\left(\tilde{J}_{2}, \tilde{J}_{6}, \tilde{J}_{10}, \tilde{J}_{15}\right)\right)
$$

$$
\begin{gathered}
A_{\mathbb{F}_{3}}\left(\Gamma_{\mathbb{K}}\right) \cong \mathbb{F}_{3}\left[\tilde{J}_{6}, \tilde{J}_{10}, \tilde{J}_{15}\right] /\left(\tilde{C}_{p}\right) \\
A_{\mathbb{F}_{2}}\left(\Gamma_{\mathbb{K}}\right) \cong \mathbb{F}_{2}\left[\tilde{J}_{6}, \tilde{J}_{10}, \tilde{J}_{12}, \tilde{J}_{15}\right] /\left(\tilde{J}_{6}^{2}+\tilde{J}_{10}^{2}, \tilde{J}_{15}^{2}+\tilde{J}_{10}^{3}+\tilde{J}_{12}^{2}\right) .
\end{gathered}
$$

\section{References}

[1] BAILY, W. L., JR.: Automorphic forms with integral Fourier coefficients. In Several Complex Variables, I (Proc. Conf., Univ. of Maryland, College Park, Md., 1970), pp. 1-8. Springer Verlag, 1970.

[2] BAILY, W. L., JR.: Theorems on the finite generation of algebras of modular forms. Amer. J. Math. 104 (1982), 645-682.

[3] Goren, E. Z.: Hilbert modular forms modulo $p^{m}$ : the unramified case. J. Number Theory 90 (2001), 341-375.

[4] Goren, E. Z.: Lectures on Hilbert modular varieties and modular forms. CRM Monograph series 14. American Mathematical Society, Providence, RI, 2002. 
[5] NagaokA, S.: On the ring of Hilbert modular forms over $\mathbb{Z}$. J. Math. Soc. Japan 35 (1983), 589-608.

[6] NagaokA, S.: On Hilbert modular forms with integral Fourier coefficients. Abh. Math. Sem. Univ. Hamburg 56 (1986), 157-168.

[7] NagaokA, S.: Note on mod $p$ Siegel modular forms. Math. Z. 235 (2000), 405-420.

[8] Serre, J.-P.: Formes modulaires et fonctions zêta p-adiques. In Modular functions of one variable, III (Proc. Internat. Summer School, Univ. Antwerp, 1972), 191-268. Lecture Notes in Math. 350. Springer Verlag, Berlin, 1973.

[9] Swinnerton-Dyer, H. P. F.: On $l$-adic representations and congruences for coefficients of modular forms. In Modular functions of one variable, III (Proc. Internat. Summer School, Univ. Antwerp, 1972), 1-55. Lecture Notes in Math. 350. Springer Verlag, Berlin, 1973.

Recibido: 19 de septiembre de 2003

Shoyu Nagaoka

Department of Mathematics

Kinki University

Higashi-Osaka

Osaka 577-8502, Japan

nagaoka@math.kindai.ac.jp 